Originalien

Chirurg 2021 - 92:1033-1039

https://doi.org/10.1007/s00104-020-01137-3

Online publiziert: 14. Februar 2020

(c) Der/die Autor(en) 2020

D. Liebetrau' ' E. Feder' ' S. Zerwes ' Y. Goßlau' · A. Hyhlik-Dürr'

'Klinik für Gefäßchirurgie und endovaskuläre Chirurgie, Universitätsklinikum Augsburg, Augsburg, Deutschland

${ }^{2}$ Klinik Günzburg, Günzburg, Deutschland

\title{
Behandlungsstrategien und Ergebnisse bei injektionsassoziierten inguinalen perivaskulären Abszessen bei intravenös Drogenabhängigen
}

Durch einen langjährigen intravenösen (i.v.) Drogenkonsum kommt es im Laufe der Zeit zu Vernarbungen im Bereich der oberflächlichen Venen. Als alternativer Zugang werden dann bevorzugt die großen, tiefliegenden Gefäße der unteren Extremität gewählt [18].

Dabei fördert die Verwendung verunreinigter, kontaminierter Injektionsnadeln bakterielle Infektionen. Ausgedehnte Abszedierungen können auftreten und gehen mit einem hohen Risiko schwerwiegender Gefäßkomplikationen einher. Die Behandlung dieser Komplikationen stellt anhaltend eine große medizinische Herausforderung dar.

\section{Zielsetzung der Arbeit}

Nach Recherchen in der medizinischen Fachliteratur existiert keine einheitliche Vorgehensweise zur Behandlung perivaskulärer Inguinalabszesse nach intravasaler Drogeninjektion. Zielsetzung dieser Arbeit ist es, einen Behandlungspfad sowie mögliche diagnostische Maßnahmen und chirurgische Therapiestrategien bei inguinalen Abszessen durch intravenösen Drogenabusus darzustellen.

\section{Patientenkollektiv und Methoden}

Im Zeitraum 01.01.2004 bis 01.05.2019 wurden alle am Universitätsklinikum Augsburg behandelten Drogenkonsu- menten mit Abszedierung der Inguinalregion in einer prospektiven Datenbank (Microsoft-Excel $^{\circledR}$-Version 2010; Microsoft Corporation, Redmond, WA, USA) erfasst und retrospektiv ausgewertet. Eingeschlossen wurden alle Patienten, die sich mit einem dokumentierten aktiven oder anamnestisch erhobenen Drogenabusus und einer Leisteninfektion vorstellten. Es konnten 37 Fälle (25 männliche, 12 weibliche Patienten) nach Anwendung der Einschlusskriterien in die Datenerhebung eingeschlossen werden.

Für die retrospektive Datenerhebung wurden die Krankenakten bzw. Mikrofilme und Operationsberichte zur Analyse herangezogen. Bei allen Patienten wurde bei jedem Aufenthalt eine virologische Diagnostik mittels HIV-Antikörpersuchtest-Diagnostik und Hepatitisserologie veranlasst. Als Komplikation wurde das Auftreten einer Blutung, eines akuten Nierenversagens, einer Nervenläsion, einer Pneumonie oder Ischämie der unteren Extremität im Rahmen des stationären Aufenthaltes definiert. Aufgrund des unterschiedlichen Infektionsausmaßes wurde das Patientenkollektiv in 4 Gruppen eingeteilt: 14 Fälle, bei denen keine Gefäßbeteiligung vorlag, 10 Fälle mit rein venöser Beteiligung, 11 Fälle mit rein arterieller und 2 Fälle mit einer kombiniert arteriovenösen Beteiligung.
Universitätsklinikum Augsburg zwischen dem 01.01.2004 und dem 01.05.2019 behandelten intravenös Drogenabhängigen mit einem inguinalen Abszess. 
Tab. 1 Patientencharakteristika

\begin{tabular}{lll}
\hline Patientenkollektiv & $\begin{array}{l}\text { Gesamt } \\
(\boldsymbol{n})\end{array}$ & $\mathbf{( \% )}$ \\
\hline Einbezogene Patienten & 37 & 100,0 \\
\hline Geschlecht (m/w) & $25 \mid 12$ & $67,6 \mid 32,4$ \\
\hline Alter in Jahren (MW \pm SD) & $35,7 \pm 7,2$ & - \\
\hline Median des Alters & 34,3 & - \\
\hline Leukozyten/nl (MW \pm SD) & $15,3 \pm 6,8$ & - \\
\hline CRP mg/dl (MW \pm SD) & $19,6 \pm 9,6$ & - \\
\hline Fieber & 27 & 73,0 \\
\hline Tage ITS (MW \pm SD) & $3,2 \pm 7,2$ & - \\
\hline HIV & 1 & 2,7 \\
\hline Hepatitis C & 25 & 67,6 \\
\hline Polytoxikomanie & 33 & 89,2 \\
\hline Alkoholabusus & 7 & 18,9 \\
\hline Nikotinabusus & 30 & 81,1 \\
\hline Dauer KH-Aufenthalt (Tage) & $35,1 \pm 21,6$ & - \\
\hline Durchgeführte Major-Amputationen & 1 & 2,8 \\
\hline MW Mittelwert, SD Standardabweichung, CRP C-reaktives Protein, ITS Intensivstation, HIV Huma- \\
\hline ne-Immundefizienz-Virus, KH Krankenhaus
\end{tabular}

Tab. 2 Zusammenfassung der behandelten perivaskulären Leistenabszesse mit arterieller Gefäßbeteiligung

\begin{tabular}{|c|c|c|}
\hline & $\begin{array}{l}\text { Primär } \\
(n)\end{array}$ & $\begin{array}{l}\text { Sekundär } \\
\text { (n) }\end{array}$ \\
\hline Einbezogene Patienten & 13 & 5 \\
\hline Ligatur der Arterie & 5 & 2 \\
\hline \multicolumn{3}{|l|}{ Interponat } \\
\hline Autolog (Vene) & 5 & 3 \\
\hline Alloplastisch & - & 1 \\
\hline Extraanatomischer Bypass & 1 & - \\
\hline \multicolumn{3}{|l|}{ Patch } \\
\hline Autolog (Vene) & 1 & - \\
\hline Bovin & 1 & - \\
\hline Ischämiesymptomatiknach arterieller Ligatur & 2 & - \\
\hline$A B I^{a}$ nach arterieller Ligatur $(M W \pm S D)$ & $0,65 \pm 0,24$ & \\
\hline Follow-up arterieller Rekonstruktionen (Tage) & 421 & \\
\hline Offenheitsrate arterieller Rekonstruktionen & $87,5 \%$ & \\
\hline
\end{tabular}

\section{Ergebnisse}

\section{Patientencharakteristika}

Das mediane Alter im untersuchten $\mathrm{Pa}$ tientenkollektiv lag bei 34,3 Jahren. Zum Zeitpunkt der Aufnahme zeigten sich im Durchschnitt eine erhöhte Leukozytenzahl von $15,3 \pm$ Standardabweichung (SD) 6,8/nl und ein C-reaktives Pro-

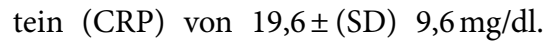
Weiterhin zeigte sich bei 20 Personen zum Zeitpunkt des Erstkontakts Fieber mit einer Durchschnittstemperatur von $38,5^{\circ} \pm(\mathrm{SD}) 0,65$. Die mittlere Aufenthaltsdauer der Patienten betrug 35,1 \pm (SD) 21,6 Tage. Die 30-Tage-Mortalität lag bei 2,7\% (1/37). Ein Patient verstarb an einem septischen Multiorganversagen. Bei einem Patienten musste aufgrund des ausgeprägten Weichteilinfektes eine Major-Amputation durchgeführt werden. Dies entspricht einer Amputationsrate von 2,8\% (•Tab. 1). Im untersuchten Kollektiv lag bei $13 \mathrm{~Pa}$ tienten eine arterielle Beteiligung vor (- Tab. 2). In 5 Fällen wurde primär arteriell ligiert. Bei weiteren 5 Fällen wurde primär eine Rekonstruktion mittels autologen Interponats durchgeführt. In weiteren 3 Fällen erfolgte die Anlage eines Obturatorbypasses (1/3) sowie die Durchführung einer Patchplastik (2/3). Zwei der 5 primär ligierten Patienten entwickelten im stationären Verlauf eine Extremitätenischämie und wurden sekundär einer Revaskularisation mittels autologen Interponats unterzogen. Insgesamt konnte bei 5/8 Patienten (arterielle Rekonstruktionen) ein Follow-up erhoben werden. Die Offenheitsrate nach arterieller Rekonstruktion lag bei 87,5\% bei einem mittleren Follow-up von 421 Tagen. Die Gesamtkomplikationsrate lag bei $51,4 \%$ (• Tab. 3 ).

\section{Diagnostischer Algorithmus}

Alle injektionsassoziierten Lokalbefunde wurden zunächst einer farbkodierten duplexsonographischen Diagnostik zur Beurteilung der Abszessformation, Gefäßbeteiligung sowie Flussbeurteilung in den Femoralgefäßen unterzogen. In 13 Fällen erfolgte eine Röntgen(RT)Übersichtsaufnahme zur Detektion eines Fremdkörpers. In 22 Fällen wurde bei nicht ausreichender Erfassung durch die Duplexsonographie aufgrund weitreichender teilweise multilokulärer inguinaler und retroperitonealer $\mathrm{Ab}$ szedierungen sowie nicht sicherer $\mathrm{Be}$ urteilung der Gefäßsituation zusätzlich eine computertomographische Angiographie (CTA; SOMATOM Definition Flash, Siemens: $3 \mathrm{~mm}$ Schichtdicke) präoperativ durchgeführt. Die Kombination verschiedener Untersuchungen war möglich. Während des stationären Aufenthaltes erfolgte bei allen Patienten die konsiliarische Mitbeurteilung durch die Kollegen der Psychiatrie und bei Bedarf die Erstellung eines individuellen Substitutionsprogramms mittels Polamidon (• Abb. 1). 
Chirurg 2021 · 92:1033-1039 https://doi.org/10.1007/s00104-020-01137-3

(c) Der/die Autor(en) 2020

D. Liebetrau · E. Feder · S. Zerwes · Y. Goßlau · A. Hyhlik-Dürr

\section{Behandlungsstrategien und Ergebnisse bei injektionsassoziierten inguinalen perivaskulären Abszessen bei intravenös Drogenabhängigen}

\section{Zusammenfassung}

Hintergrund. Langzeitdrogenabhängige präsentieren sich nach dem Verbrauch der oberflächlichen Venen regelmäßig mit tiefen inguinalen vaskulär assoziierten Abszessen infolge fortgesetzter Drogeninjektionen. Die Behandlung dieser Komplikationen stellt anhaltend eine große medizinische Herausforderung dar. Bisher werden keine einheitlichen Therapieregime in der Literatur beschrieben.

Fragestellung. Welche Behandlungsstrategien und Ergebnisse gibt es bei injektionsassoziierten inguinalen perivaskulären Abszessen bei Drogenabhängigen? Material und Methoden. Die Daten aller im Zeitraum zwischen 01.01.2004 und 31.05.2019 am Universitätsklinikum Augsburg behandelten Drogenkonsumenten wurden retrospektiv aufgearbeitet und mit der vorliegenden Literatur verglichen.

Ergebnisse. Es konnten 37 Fälle (männlich $=25$, weiblich $=12$ ) nach Anwendung der Einschlusskriterien in die Datenerhebung eingeschlossen werden. Das mediane Alter im untersuchten Patientenkollektiv lag bei 34,3 Jahren. Die 30-Tage-Mortalität lag bei 2,7\% (1/37). Die Amputationsrate betrug $2,8 \%$. Im untersuchten Kollektiv lag bei 13 Patienten eine arterielle Beteiligung vor. In 5 Fällen wurde primär arteriell ligiert. Bei weiteren 5 Fällen wurde primär eine Rekonstruktion mittels autologen Interponats durchgeführt. In weiteren 3 Fällen erfolgte die Anlage eines Obturatorbypasses (1/3) sowie die Durchführung einer Patchplastik (2/3). Die Offenheitsrate nach arterieller Rekonstruktion lag bei $87,5 \%$ bei einem mittleren Follow-up von 421 Tagen. Die Gesamtkomplikationsrate lag bei $51,4 \%$.

Diskussion. Bei vaskulärer Beteiligung ist ein situationsgerechtes Vorgehen sinnvoll. Neben der Beseitigung komplizierter, septischvenöser Thrombosen erscheint die Korrektur arterieller Blutungen mittels autologer Rekonstruktionsmaßnahmen aussichtsreich.

\section{Schlüsselwörter}

Leisteninfektion · Intravenöser Drogenabusus . Septische Arrosionsblutung · Leistenabszess . Gefäßkomplikation

\section{Treatment strategies and outcomes for injection-associated inguinal perivascular abscesses in intravenous drug addicts}

\section{Abstract}

Background. Long-term drug users regularly present with deep inguinal vascularassociated abscesses due to continued drug injections utilizing superficial veins. The treatment of these complications continues to be a major medical challenge. So far no uniform treatment regimens have been described in the literature.

Objective. What are the treatment strategies and outcomes of injection-associated inguinal perivascular abscesses in drug addicts? Material and methods. All drug users treated at the Augsburg University Hospital in the period between 1 January 2004 and 31 May 2019 were retrospectively reviewed and compared with the currently available literature.

Results. In this study 37 cases (male $=25$, female $=12$ ) could be included in the data collection after implementation of the inclusion criteria. The median age in the investigated patient population was 34.3 years. The 30 -day mortality was $2.7 \%$ $(1 / 37)$. The amputation rate was $2.8 \%$. In the investigated collective 13 patients had arterial involvement, in 5 cases a ligature of arteries was primarily used and in another 5 cases a reconstruction using an autologous conduit graft was primarily performed. In another 3 cases an obturator bypass (1/3) was placed and a patch plasty $(2 / 3)$. The patency rate after arterial reconstruction was $87.5 \%$ with a mean follow-up of 421 days. The overall complication rate was $51.4 \%$.

Conclusion. For vascular involvement an approach appropriate for the situation is meaningful. In addition to the elimination of complicated septic venous thromboses, the correction of arterial hemorrhages using autologous reconstruction measures seems promising.

\section{Keywords}

Groin infection - Intravenous drug abuse . Septic arrosion bleeding - Groin abscess . Vascular complication

\section{Diskussion}

Die Altersverteilung lag in der zu erwartenden Spannbreite zwischen 22 und 50 Jahren. Es wurden mit $67,7 \%$ deutlich häufiger Männer als Frauen mit dem komplexen Krankheitsbild eines injektionsassoziierten Leistenabszess behandelt. Dies korreliert mit den Angaben der Rauschgiftdatei des Bundeskriminalamts von 2017, indem die registrierten Drogentoten bei einem Durchschnitts- alter von 39 Jahren zu $85 \%$ männlich waren [3]. Ein Patient verstarb während des stationären Aufenthaltes und bei nur einem Patienten musste eine Major-Amputation durchgeführt werden. In unserem Patientenkollektiv konnte in $97,2 \%(35 / 36)$ ein amputationsfreies Überleben erreicht werden. Insgesamt ergab sich trotz des jungen Patientenalters eine durchschnittliche stationäre Verweildauer von $35,1 \pm 21,6$ Tagen. Die hohe Gesamtkomplikationsrate von
$51,4 \%$ unterstreicht die hohe Morbidität der inguinalen Weichteilinfektionen.

\section{Präoperative Diagnostik}

Auffällig ist, dass in den primär durchgeführten duplexsonographischen Befunden in keinem Fall der Verdacht auf das Vorliegen eines Spritzenfragmentes beschrieben wurde. Es konnte jedoch in insgesamt 4 Kasuistiken ein abgebrochenes Nadelfragment radiologisch nachge- 


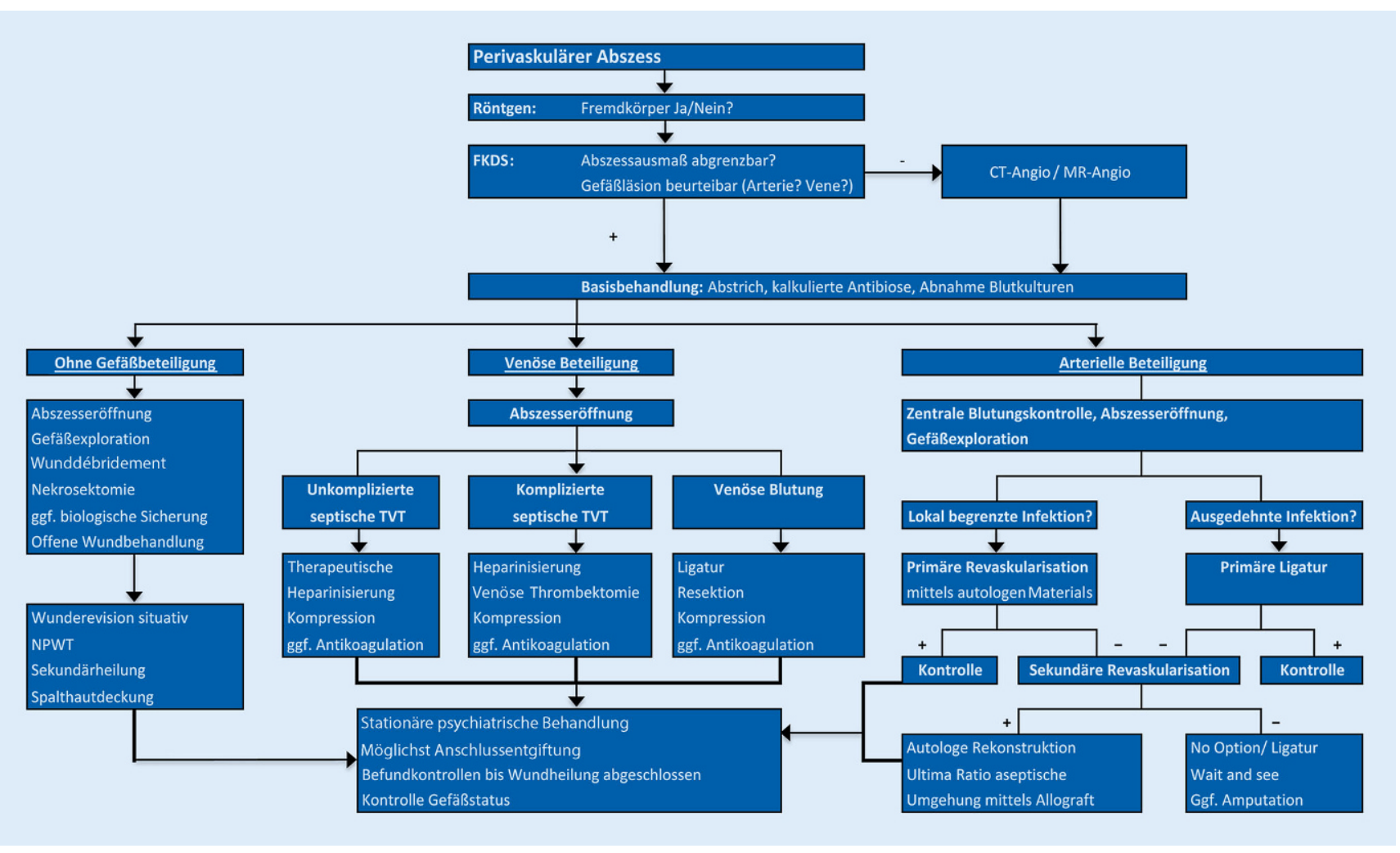

Abb. 1 А Diagnostik- und Therapiealgorithmus bei perivaskulären Abszessen. NPWT "negative pressure wound therapy", TVT tiefe Beinvenenthrombose, FKDS farbkodierte Duplexsonographie, CT-Angio computertomographische Angiographie, MR-Angio Magnetresonanzangiographie

wiesen werden. Drei wurden dabei durch eine Beckenübersichtsaufnahme und eine primär in der CT-Angiographie diagnostiziert. Die Fremdkörper wurden intraoperativ unter Durchleuchtung geborgen. Die explizite Abklärung diesbezüglich ist für den Eigenschutz unabdingbar und besitzt höchste Priorität. Eine Untersuchung aus Großbritannien konnte zeigen das $20 \%$ der i.v. Drogenabhängigen Erfahrungen mit abgebrochenen Nadeln gemacht haben [16]. Daher sollte zwingend ein radiologischer Ausschluss von Fremdkörpern zum Eigenschutz erfolgen (• Abb. 1).

\section{Operative Ergebnisse}

Bei der Behandlung komplizierter Inguinalabszesse mit Beteiligung der Femoralgefäße ist in der Literatur kein einheitliches Therapiekonzept beschrieben. Als allgemein gültige Strategie bei der Behandlung von Spritzenabszessen wird die ausgedehnte chirurgische Infektspaltung mit Abszesseröffnung und weitreichendem Weichteildébridement sowie anschließender konsequenter offener Wundbehandlung akzeptiert [9, 19, 22]. Die relativ kleine Fallzahl von 37 Inguinalabszessen ergibt sich daraus, dass keine oberflächlichen bzw. subkutanen Abszedierungen, sondern nur komplikationsträchtige Infektionen, die bis zu den Femoralgefäßen reichten, untersucht wurden. Verglichen mit anderen Veröffentlichungen $[2,9,13,14]$ ist das in dieser Arbeit vorgestellte Patientenkollektiv mit insgesamt 37 Fällen eines der größten deutschen untersuchten Kollektive.

Grundsätzlich zeigten sich im untersuchten Kollektiv 4 unterschiedliche Beteiligungsmuster der Femoralgefäße (- Tab.3). Dabei kommen unterschiedliche Behandlungsstrategien zum Tragen. Das therapeutische Vorgehen richtete sich bei Patienten mit rein venöser Beteiligung neben der chirurgische Infektspaltung nach der klinischen Beherrschbarkeit des lokalen Infektes (•Tab. 4). Bei unkomplizierten Becken-Bein-Venenthrombose erfolgte die Therapie leitliniengerecht mittels therapeutischer
Heparinisierung und Kompressionstherapie [2]. Die erfolgreiche Therapie der komplizierten/septischen iliakofemoralen Phlebothrombose kann häufig durch alleinige Heparinisierung und testgerechte Antibiotikatherapie erzielt werden [11]. In unserem untersuchten Kollektiv konnte in 6 Fällen ein konservatives Prozedere bei septischer Phlebothrombose erfolgreich durchgeführt werden. In weiteren 6 Fällen war unter konservativer Therapie keine Beherrschung des Infektgeschehens möglich. Es erfolgte daraufhin die chirurgische Therapie mittels Thrombektomie der V. femoralis communis (VFC, 1/6), Crossektomie der V. saphena magna mit Thrombektomie der VFC (1/6) oder Ligatur der VFC (4/6; - Tab. 4). Die Thrombektomie der VFC wurde in der Literatur bei fortschreitender septischer oder zentraler Thrombosierung, multifokaler Abszedierung oder Embolisation bereits beschrieben [10]. Da die operative Behandlung der septischen Thrombose mittels offener venöser Thrombektomie äußerst komplikationsträchtig ist, 
Tab. 3 Operative Behandlung und Ergebnisse bei perivaskulären Leistenabszessen

\begin{tabular}{|c|c|c|c|c|c|c|c|c|c|c|}
\hline \multirow[t]{2}{*}{$\begin{array}{l}\text { Patienten- } \\
\text { kollektiv }\end{array}$} & \multicolumn{2}{|c|}{ Gesamt } & \multicolumn{2}{|c|}{$\begin{array}{l}\text { Ohne Ge- } \\
\text { fäßbeteili- } \\
\text { gung }\end{array}$} & \multicolumn{2}{|c|}{$\begin{array}{l}\text { Venöse } \\
\text { Gefäßbetei- } \\
\text { ligung }\end{array}$} & \multicolumn{2}{|c|}{$\begin{array}{l}\text { Arterielle } \\
\text { Gefäßbetei- } \\
\text { ligung }\end{array}$} & \multicolumn{2}{|c|}{$\begin{array}{l}\text { Arterielle + } \\
\text { venöse Gefäß- } \\
\text { beteiligung }\end{array}$} \\
\hline & $(n)$ & (\%) & (n) & $(\%)$ & (n) & (\%) & (n) & (\%) & (n) & $(\%)$ \\
\hline $\begin{array}{l}\text { Einbezogene } \\
\text { Patienten }\end{array}$ & 37 & 100 & 14 & 37,8 & 10 & $27,0 €$ & 11 & 29,7 & 2 & 5,4 \\
\hline NPWT & 29 & - & 11 & 78,6 & 8 & 80,0 & 8 & 72,7 & 2 & 100,0 \\
\hline Nadelfragmente & 4 & - & 1 & 7,1 & 1 & 0,0 & 1 & 9,1 & 1 & 50,0 \\
\hline Revisionen & 111 & - & 27 & 24,3 & 15 & 13,5 & 54 & 48,6 & 15 & 13,5 \\
\hline $\begin{array}{l}\text { Biologische Siche- } \\
\text { rung }\end{array}$ & 9 & - & 2 & 14,3 & 0 & 0,0 & 6 & 54,5 & 1 & 50,0 \\
\hline Spalthautdeckung & 10 & - & 3 & 21,4 & 2 & 20,0 & 4 & 36,4 & 1 & 50,0 \\
\hline Primäre Hautnaht & 1 & - & 0 & 0,0 & 1 & 10,0 & 0 & 0,0 & 0 & 0,0 \\
\hline Sekundärnaht & 7 & - & 2 & 14,3 & 3 & 30,0 & 1 & 9,1 & 1 & 50,0 \\
\hline Sekundärheilung & 19 & - & 9 & 64,3 & 4 & 40,0 & 6 & 54,5 & 0 & 0,0 \\
\hline Komplikationen & 19 & 51,4 & 3 & 21,4 & 4 & 40,0 & 10 & 90,9 & 2 & 100,0 \\
\hline Blutung & 7 & - & 1 & 7,1 & 2 & 20,0 & 4 & 36,4 & 0 & 0,0 \\
\hline ANV & 3 & - & 1 & 7,1 & 0 & 0,0 & 2 & 18,2 & 0 & 0,0 \\
\hline Pneumonie & 4 & - & 1 & 7,1 & 1 & 10,0 & 1 & 9,1 & 1 & 50,0 \\
\hline Nervenläsion & 3 & - & 0 & 0,0 & 1 & 10,0 & 2 & 18,2 & 0 & 0,0 \\
\hline Ischämie $^{a}$ & 2 & - & 0 & 0,0 & 0 & 0,0 & 1 & 9,1 & 1 & 50,0 \\
\hline $\begin{array}{l}\text { Polamidon } \\
\text { (ml/Tag) }^{\mathrm{b}}\end{array}$ & 7,9 & - & 7,4 & - & 7 & - & 8,5 & - & 12 & - \\
\hline Beinerhalt bei & 36 & - & 14 & 38,9 & 10 & 27,8 & 10 & 27,8 & 2 & 5,6 \\
\hline \multicolumn{11}{|c|}{$\begin{array}{l}\text { ANV akutes Nierenversagen, NPWT "negative pressure wound therapy" } \\
\text { "Untere Extremitätenischämie } \\
\text { 'Durchschnittlicher Verbrauch pro Tag }\end{array}$} \\
\hline
\end{tabular}

sollte sie nur bei nicht beherrschbarer Sepsis erfolgen [7]. Weiterhin konnte eine hohe Komplikationsrate (90,9\%) in der Gruppe der Leistenabszesse mit arterieller Gefäßbeteiligung erhoben werden. Eine Möglichkeit, welche mit einem geringen Komplikationsrisiko hinsichtlich nachfolgender septischer Arrosionsblutung und postoperativer Letalität behaftet ist, stellt die primäre Ligatur der Leistenarterien dar. Dies gilt vor allem für isolierte Gefäßläsionen unterhalb der Femoralisgabel im Bereich der A. femoralis superficialis und A. profunda femoris, da meist eine ausreichende Kollateralisation über proximale Gefäßabschnitte vorhanden ist. Es gibt verschiedene Arbeiten, in denen die primäre Ligatur der Iliakalgefäße und der A. femoralis communis als erste Therapie der Wahl herangezogen wurde. Dabei wurde das amputationsfreie Überleben mit bis zu $99 \%$ angegeben [15, 19]. Im untersuchten Patientenkollektiv wurde die primäre Ligatur bei 5 von
13 Patienten mit arterieller Gefäßbeteiligung durchgeführt (•Tab. 2). Zwei der 5 Patienten entwickelten im stationären Verlauf eine Extremitätenischämie und wurden einer Revaskularisation mittels Veneninterponat unterzogen. In der Literatur wurde dieses Vorgehen bei amputationsbedrohter Extremität ebenfalls berichtet $[8,14,17]$. Bei den übrigen primär ligierten Patienten lag der durchschnittliche Ankle-brachial-Index (ABI) bei $0,65 \pm 0,24$. Trotz klinisch kompensierter Hämodynamik sollte eine Ligatur aus unserer Sicht bei einem durchschnittlichen $\mathrm{ABI}$ von 0,65 nicht primär favorisiert werden.

Um einem ischämischen Verlauf und konsekutiven Extremitätenverlust vorzubeugen, favorisieren wir, wie auch andere Autoren, wenn möglich die primäre Revaskularisation des resezierten septischen Gefäßabschnitts [5, 6, 9, 22]. In unserem Patientenkollektiv erfolgte in 5 Fällen primär die arterielle Rekonstruktion progredienter Ischämiesymptomatik mit
Tab. 4 Zusammenfassung der behandel-

ten inguinalen septischen Thrombosen

\begin{tabular}{|c|c|}
\hline & $\begin{array}{l}\text { Primär } \\
n\end{array}$ \\
\hline Einbezogene Patienten & 12 \\
\hline Ligatur der Vene $^{\mathrm{a}}$ & 4 \\
\hline \multicolumn{2}{|l|}{ Thrombektomie } \\
\hline Singulär & 1 \\
\hline Zusätzlich Crossektomie & 1 \\
\hline Konservativ ${ }^{\mathrm{b}}$ & 6 \\
\hline \multicolumn{2}{|c|}{$\begin{array}{l}\text { aLigatur der V. iliaca/femoralis communis/ } \\
\text { femoralis profunda oder femoralis superfi- } \\
\text { cialis } \\
{ }^{b} \text { Mittels therapeutischer Heparinisierung } \\
\text { und testgerechter Antibiose } \\
\text { 'Primäre Versorgung bei venöser Gefäßbe- } \\
\text { teiligung }\end{array}$} \\
\hline
\end{tabular}

unter Verwendung der ipsilateralen V. femoralis communis. In 2 Fällen wurde primär eine Patchplastik, einmal autologe Vene und einmal bovines Rinderperikard (XenoSure ${ }^{\circledast}$, LeMaitre Vascular, Inc., Burlington, USA), auf das arrodierte Arteriensegment angelegt. In beiden Fällen kam es im Verlauf zu einer septischen Arrosionsblutung mit Patchausriss. Bei dem Patient mit Venenpatchplastik erfolgte in einer sekundären Operation die Rekonstruktion mittels V. femoralis communis, im anderen Fall bei fehlender geeigneter autologer Vene die arterielle Rekonstruktion mittels Allograft (MAQUET, INTERGARD SYNERGY, Durchmesser $8 \mathrm{~mm}$ ). Grundsätzlich kann festgehalten werden, dass die Verwendung eines Patches auf Höhe des geschädigten Gefäßsegmentes in unserer Untersuchung nicht langfristig erfolgreich war (2 Blutungen bei 2 Anlagen) und das Risiko einer weiterführenden septischen Arrosion in dem betroffenen Segment hoch und nicht Erfolg versprechend ist.

Bei einem Patienten trat nach primärer Rekonstruktion durch Veneninterponat bei fortschreitendem Infektgeschehen eine septische Arrosionsblutung des autologen Graftmaterials auf. Hier musste eine sekundäre Ligatur auf Höhe der A. iliaca externa mit Resektion des Bypasses vorgenommen werden. Es zeigte sich hinterher keine kritische Extremitätenischämie.

Als Alternative zum autologen Gefäßersatz können extraanatomisch ver- 
laufende Bypässe (z. B. Obturatorbypass), unter Umgehung des lokalen Infektes, angelegt werden $[12,20]$. In unserem Kollektiv wurde primär bei einem Patienten ein alloplastischer Obturatorbypass angelegt. Dabei konnte ein Verschluss nach 220 Tagen festgestellt werden. Insgesamt konnte von den arteriellen Rekonstruktionen bei 5 von 8 Patienten ein Follow-up bestimmt werden. Die Offenheitsrate der gesamten arteriellen Rekonstruktionen betrug $87,5 \%$ bei einem mittleren Follow-up von 421 Tagen. Dabei waren alle venösen Interponate im untersuchten Zeitraum perfundiert. Lediglich der Obturatorbypass zeigte sich verschlossen. In der Literatur werden Offenheitsraten von Obturatorbypässen mit $65 \%$ bei einem Follow-up von 24 Monaten angegeben [4]. Grundsätzlich besteht bei nur einem angelegten Obturatorbypass keine suffiziente Aussagekraft, jedoch lässt sich in Zusammenschau der erhobenen Daten eine Tendenz zugunsten der autologen Gefäßrekonstruktion feststellen. Hier sind weitere Untersuchungen notwendig. Weiterhin sind alloplastische Gefäßrekonstruktionen aufgrund der niedrigen Compliance von Drogenpatienten durch erneute Injektionen bei fortgesetztem Drogenkonsum stark infektgefährdet [23]. Dieses Risiko ist bei den extraanatomischen Rekonstruktionen hoch, da diese bei subkutanen Verläufen leicht für weiterführende Drogeninjektionen missbraucht werden können [21]. Aus unserer Sicht sollte die Anlage eines alloplastischen extraanatomischen Bypass nur selektiv und übergangsweise zur sekundären Revaskularisation bei kritischer Extremitätenischämie erfolgen. In der Literatur wurde dieses Vorgehen zur Senkung der Amputationsrate bereits beschrieben [13]. Schlussendlich erscheint bei einer festgestellten Offenheitsrate von $100 \%$ die Korrektur arterieller Blutungen unter Anlage eines autologen Interponats als aussichtsreich.

\section{Fazit für die Praxis}

\section{- Nach Differenzierung des Lokalbe-} fundes (FKDS, CTA) ist unter kalkulierter Antibiose ( $\beta$-Laktamasestabil) eine breite Abszesseröffnung mit Exploration der Inguinalgefäße und offener Wundbehandlung durch einen in der Gefäßmedizin erfahrenen Chirurgen als Basismaßnahme erforderlich.

- Bei der Wundexploration ist im Sinne des Eigenschutzes auf den Ausschluss von Nadelfragmenten zu achten.

- Bei vaskulärer Beteiligung ist ein situationsgerechtes Vorgehen sinnvoll. Neben der Beseitigung komplizierter, septisch-venöser Thrombosen erscheint die Korrektur arterieller Blutungen mittels autologer Rekonstruktionsmaßnahmen aussichtsreich.

- Aufgrund der guten Kollateralisation der im Grunde gefäßgesunden Patienten ist als Ultima Ratio eine primäre Ligatur in Sondersituationen vertretbar.

\section{Korrespondenzadresse}

\section{Dr. med. D. Liebetrau}

Klinik für Gefäßchirurgie und endovaskuläre Chirurgie, Universitätsklinikum Augsburg Stenglinstraße 2, 86156 Augsburg, Deutschland dominik.liebetrau@uk-augsburg.de

Funding. Open Access funding provided by Projekt DEAL.

\section{Einhaltung ethischer Richtlinien}

Interessenkonflikt. D. Liebetrau, E. Feder, S. Zerwes, Y. Goßlau und A. Hyhlik-Dürr geben an, dass kein Interessenkonflikt besteht.

Alle beschriebenen Untersuchungen am Menschen oder an menschlichem Gewebe wurden mit Zustimmung der zuständigen Ethikkommission, im Einklang mit nationalem Recht sowie gemäß der Deklaration von Helsinki von 1975 (in der aktuellen, überarbeiteten Fassung) durchgeführt. Von allen beteiligten Patienten liegt eine Einverständniserklärung vor.

Open Access. Dieser Artikel wird unter der Creative Commons Namensnennung 4.0 International Lizenz veröffentlicht, welche die Nutzung, Vervielfältigung, Bearbeitung, Verbreitung und Wiedergabe in jeglichem Medium und Format erlaubt, sofern Sie den/die ursprünglichen Autor(en) und die Quelle ordnungsgemäß nennen, einen Link zur Creative Commons Lizenz beifügen und angeben, ob Änderungen vorgenommen wurden.

Die in diesem Artikel enthaltenen Bilder und sonstiges Drittmaterial unterliegen ebenfalls der genannten Creative Commons Lizenz, sofern sich aus der Abbildungslegende nichts anderes ergibt. Sofern das betreffende Material nicht unter der genannten Creative Commons Lizenz steht und die betreffende Handlung nicht nach gesetzlichen Vorschriften erlaubt ist, ist für die oben aufgeführten Weiterverwendungen des Materials die Einwilligung des jeweiligen Rechteinhabers einzuholen.

Weitere Details zur Lizenz entnehmen Sie bitte der Lizenzinformation auf http://creativecommons.org/ licenses/by/4.0/deed.de.

\section{Literatur}

1. https://wdr.unodc.org/wdr2019/.Zugegriffen: 26 . Juni 2019

2. https://www.awmf.org/uploads/tx_szleitlinien/ 065-002k_S2k_VTE_VenenthromboseLungenembolie_2017-04.pdf. Zugegriffen: 4. Okt. 2019

3. https://www.bka.de/SharedDocs/Downloads/ DE/Publikationen/JahresberichteUndLagebilder/ Rauschgiftkriminalitaet/

2017RauschgiftBundeslagebildZ.html?nn=27972 Zugegriffen: 1.Okt. 2019

4. Bath J, Rahimi M, Long B et al (2017) Clinical outcomes of obturator canal bypass. J Vasc Surg 66:160-166

5. Ben Hammamia M, Fourati M, Ziadi J et al (2018) Femoral pseudoaneurysms in drug addicts: About 4 cases. JMed Vasc 43:193-197

6. Georgiadis GS (2007) Regarding "Infected femoral artery pseudoaneurysm in drug addicts: The beneficial use of the internal iliac artery for arterial reconstruction".J Vasc Surg 46:613

7. Golovyan D, Wysokinski W, Mcbane RD 2nd (2012) Septic venous thromboembolism. Vasc Med 17:429-430

8. Hu ZJ, Wang SM, Li XX et al (2010) Tolerable hemodynamic changes after femoral artery ligation for the treatment of infected femoral artery pseudoaneurysm. Ann Vasc Surg 24:212-218

9. Kaiser MM, Kujath P, Muller G et al (1997) Treatment strategy in inguinal injection abscess and complications. Chirurg 68:1029-1034

10. Kujath P, Kujath C (2010) Complicated skin, skin structure and soft tissue infections-are we threatened by multi-resistant pathogens? Eur J Med Res 15:544-553

11. Mackenzie AR, Laing RB, Douglas JG et al (2000) High prevalence of iliofemoral venous thrombosis with severe groin infection among injecting drug users in North East Scotland: successful use of low molecular weight heparin with antibiotics. Postgrad Med J 76:561-565

12. Manekeller STRH, Schroeder S, Lauschke $H$, Remig J, Hirner A (2004) Analysis of Vascular Complications in Intra-Venous Drug Addicts after Puncture of Femoral Vessels. Zentralblatt Chirurgie 129(1):21-28

13. Matoussevitch V,AleksicM, Gawenda Metal(2007) Primary extraanatomical revascularization for groin infections in drug addicts. Vasa 36:210-214

14. Moini M, Rasouli MR, Rayatzadeh $\mathrm{H}$ et al (2008) Management offemoralarterypseudo-aneurysms in Iran: a single centre report of 50 cases. Acta Chir Belg 108:226-230

15. Naqi SA, Khan HM, Akhtar S et al (2006) Femoral pseudoaneurysm in drug addicts-excision without revascularization is a viable option. Eur J Vasc Endovasc Surg 31:585-587

16. Norfolk GA, Gray SF (2003) Intravenous drug users and broken needles - a hidden risk? Addiction 98:1163-1166 
17. Padberg F Jr., Hobson R 2nd, Lee B et al (1992) Femoral pseudoaneurysm from drugs of abuse: ligation or reconstruction? JVasc Surg 15:642-648

18. Pieper B, Kirsner RS, Templin TN et al (2007) Injection drug use: an understudied cause of venous disease. Arch Dermatol 143:1305-1309

19. Qiu J, Zhou W, Zhou W et al (2016) The Treatment of Infected Femoral Artery Pseudoaneurysms Secondary to Drug Abuse: 11 Years of Experience at a Single Institution. Ann Vasc Surg 36:35-43

20. Rabbani A, Moini M, Rasouli MR (2008) Obturator bypass as an alternative technique for revascularization in patients with infected femoral pseudoaneurysms. Arch Iran Med 11:50-53

21. Salehian MT, Shahid N, Mohseni M et al (2006) Treatment of infected pseudoaneurysm in drug abusers: ligation or reconstruction? Arch Iran Med 9:49-52

22. Scheuerlein $H$, Ruff $S$, Haage $P$ et al (2008) Groin abscesses and vascular catastrophes in intravenous drug users-strategy and results. ZentralblChir 133:55-60

23. Welch GH, Reid DB, Pollock JG (1990) Infected false aneurysms in the groin of intravenous drug abusers. BrJ Jurg 77:330-333

\section{DEUTSCHER KREBSKONGRESS}

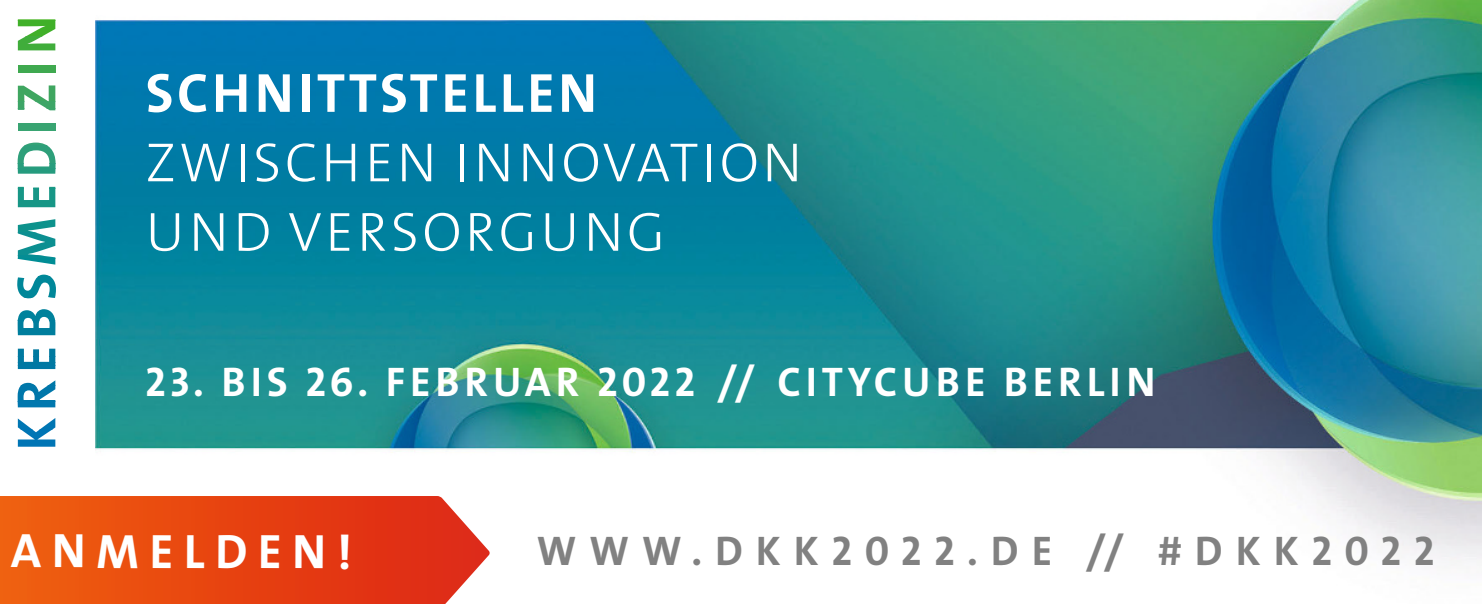

